TRANSACTIONS OF THE

AMERICAN MATHEMATICAL SOCIETY

Volume 352, Number 3, Pages 1131-1147

S 0002-9947(99)02531-3

Article electronically published on September 17, 1999

\title{
EMBEDDINGS IN GENERALIZED MANIFOLDS
}

\author{
J. L. BRYANT AND W. MIO
}

\begin{abstract}
We prove that a $(2 m-n+1)$-connected map $f: M^{m} \rightarrow X^{n}$ from a compact PL $m$-manifold $M$ to a generalized $n$-manifold $X$ with the disjoint disks property, $3 m \leq 2 n-2$, is homotopic to a tame embedding. There is also a controlled version of this result, as well as a version for noncompact $M$ and proper maps $f$ that are properly $(2 m-n+1)$-connected. The techniques developed lead to a general position result for arbitrary maps $f: M \rightarrow X$, $3 m \leq 2 n-2$, and a Whitney trick for separating $P L$ submanifolds of $X$ that have intersection number 0 , analogous to the well-known results when $X$ is a manifold.
\end{abstract}

\section{INTRODUCTION}

The recent discovery of exotic generalized manifolds in dimensions $>5$ opened a number of questions about the geometric topology of these spaces [3]. Previously conjectured by Cannon [4] to be topological manifolds if they satisfied a minimal general position property known as the disjoint disks property $(D D P)$, these spaces may, in fact, fail to be topological manifolds in a very strong sense. In [13, 14], Quinn associated to any connected generalized $n$-manifold, $n \geq 4$, a local index $\imath(X) \in 1+8 \mathbb{Z}$, which, combined with Edwards' CE-approximation theorem [6], leads to the celebrated Edwards-Quinn characterization of topological manifolds: if $n \geq 5$, a generalized $n$-manifold with the DDP is a topological manifold if and only if $\imath(X)=1$. In [3], generalized $n$-manifolds having arbitrary local index are constructed in all dimensions $>5$.

Quinn's work $[13,14]$ on the characterization of topological manifolds also bridges important decomposition theoretic aspects of manifold theory to the surgery classification of manifolds [19]. As pointed out by Cappell, closed generalized $n$-manifolds with non-unitary local index naturally correct structural anomalies observed in the surgery classification of manifolds, namely, the failure of Siebenmann periodicity for closed manifolds $[11,12]$. This strongly supports the viewpoint that the class of manifolds should be expanded to include these newly discovered spaces, but difficult questions concerning the extent to which they resemble topological manifolds have to be answered before a truly convincing case can be made.

This paper is part of our program for studying the local-global topology of generalized manifolds in higher dimensions. Using decomposition theory methods, we extend embedding techniques from $P L$ topology to the case in which the ambient space $X^{n}$ is a generalized $n$-manifold with the disjoint disks property, $n \geq 5$. More

Received by the editors December 4, 1997.

2000 Mathematics Subject Classification. Primary 57N35; Secondary 57P99.

Key words and phrases. Generalized manifolds, embeddings.

Partially supported by NSF grant DMS-9626624. 
specifically, we establish analogues of Hudson's codimension three embedding theorem [9] for embeddings of $P L$ manifolds $M^{m} \hookrightarrow X^{n}$. We will present proofs for embeddings in the metastable range, $3 m \leq 2 n-2$.

Theorem 1.1. Suppose that $X^{n}, n \geq 5$, is a generalized $n$-manifold with the disjoint disks property, $M^{m}$ is a compact $P L$ m-manifold, $3 m \leq 2 n-2$, and $f: M \rightarrow X$ is a $(2 m-n+2)$-connected map, such that $f \mid \partial M$ is $(2 m-n)$-connected. Then $f$ is homotopic to a $1-L C C$ embedding $f^{\prime}: M \rightarrow X$. Moreover, if $f \mid \partial M$ is an embedding, then we can get $f^{\prime}|\partial M=f| \partial M$.

The methods employed in the proof of the theorem also allow us to remove certain shortcomings in the general position results of [2] and [20]. Cannon observed early on that the disjoint disks property was sufficient to guarantee that any map of a 2complex into a generalized $n$-manifold with the $D D P, n \geq 5$, could be approximated by embeddings [5]. Bryant [2] and Walsh [20] were able to establish general position theorems for maps of arbitrary polyhedra (or compacta) in the sense that maps of any two such objects can be approximated by maps whose images intersect in the dimension predicted by general position. In particular, one could approximate a map of a $k$-dimensional polyhedron into a generalized $n$-manifold with the $D D P$ by an embedding, provided that $2 k+1 \leq n$. Once this dimensional restriction is relaxed, however, the results of [2] and [20] have an unfortunate shortcoming: although the singular set of a map of a $k$-dimensional polyhedron or compactum into a $D D P$ generalized $n$-manifold can be made to have dimension at most $2 k-n$, the singular set might be dense in the domain. This can have a severe dampening effect on attempts to reproduce inductive arguments that one is accustomed to exploiting in $P L$ topology. Here, we recover general position properties of the type satisfied by mappings into a $P L$ manifold (Theorem 5.2).

We also obtain a controlled version of the above result, with a view toward the study of the local topology of generalized manifolds.

Theorem 1.2. Suppose that $X^{n}, n \geq 5$, is a generalized $n$-manifold with the disjoint disks property. For every $\epsilon>0$ there exist $\delta>0$ and $\eta>0$ such that if $M^{m}$ is a compact $P L$ m-manifold, $3 m \leq 2 n-2$, and $f: M \rightarrow X$ is a map such that $f$ is $(\delta, 2 m-n+2)$-connected over $X$ and $f \mid \partial M$ is $(\eta, 2 m-n)$-connected over $X$, then $f$ is $\epsilon$-homotopic to a 1 -LCC embedding. Moreover, if $f \mid \partial M$ is an embedding, then we can get $f^{\prime}|\partial M=f| \partial M$.

In passing, we obtain a version of the Whitney Trick, a geometric construction of basic importance in classical surgery theory, for removing intersections of manifolds of complementary dimensions and intersection number 0 in a generalized manifold with the DDP (Theorem 5.4).

Although we state the theorems for embeddings of compact manifolds, the techniques work just as well for closed mappings of noncompact manifolds, provided proper connectivity assumptions are made. In the noncompact versions $\epsilon$ and $\delta$ are replaced by positive functions on $X$.

As a corollary to Theorem 1.2 we get a version of Štan'ko's 1-LCC approximation theorem for embeddings in generalized manifolds [18].

Corollary 1.3. Suppose that $f: M \rightarrow X$ is a topological embedding of a $P L m$ manifold $M$ into a generalized $n$-manifold $X, n \geq 5$, with the disjoint disks property, $3 m \leq 2 n-2$. Then $f$ is approximable by 1-LCC embeddings. Moreover, if $f \mid \partial M$ is a 1-LCC embedding then the approximations can be made to agree with $f$ on $\partial M$. 


\section{Definitions AND PRELIMINARY RESUlts}

All spaces are metric spaces, and the terms map and mapping shall be synonymous with continuous function. A compact ANR $X$ admits a contractibility function $T:\left(0, r_{0}\right) \rightarrow(0, \infty)$ having the properties that $\lim _{r \rightarrow 0} T(r)=0, T(r) \geq r$, and metric balls of radius $<r$ homotopically contract to a point inside balls of radius $<T(r)$.

If $g: A \rightarrow B$ is a map, realize the mapping cylinder $C_{g}$, as usual, as the quotient:

$$
C_{g}=A \times I \cup B / \sim,
$$

where $(x, 1) \sim g(x)$. The mapping cylinder projection $\gamma: C \rightarrow B$ is defined by $\gamma(x, t)=g(x)$ if $x \in A$ and $\gamma(y)=y$ if $y \in B$. If $S$ is a subset of $A$, then the reduced mapping cylinder $C_{g}(\bmod S)$ is the quotient of $C_{g}$ in which $(x, t) \sim g(x)$ for all $t \in I$ when $x \in S$.

\section{Definition 2.1.}

(i) A map $f: Y \rightarrow X$ is $k$-connected if $\pi_{i}\left(C_{f}, Y\right)=0$ for $0 \leq i \leq k$. In particular, this implies that if $(Q, P)$ is a relative $k$-complex (i.e., $\operatorname{dim}(Q \backslash P) \leq k)$, then any map $\alpha:(Q, P) \rightarrow\left(C_{f}, Y\right)$ is homotopic, rel $\alpha \mid P$ to a mapping into $Y$.

(ii) Given $\epsilon>0, f$ is said to be $(\epsilon, k)$-connected if there exists $\delta>0$ such that for each relative $k$-complex $(Q, P)$ and map $\alpha:(Q, P) \rightarrow\left(N_{\delta}, Y\right)$, where $N_{\delta}$ is the $\delta$-neighborhood of $Y \times I$ in $C_{f}, \alpha$ is $\epsilon$-homotopic over $X$, rel $\alpha \mid P$, to a mapping into $Y$.

A generalized $n$-manifold ( $n$-gm) $X$ is a locally compact, (finite dimensional) euclidean neighborhood retract (ENR) such that for each $x \in X, H_{k}(X, X \backslash\{x\} ; \mathbb{Z}) \cong$ $H_{k}\left(\mathbb{R}^{n} ; \mathbb{R}^{n} \backslash\{0\} ; \mathbb{Z}\right)$. ( $\mathbb{R}^{n}$ denotes euclidean $n$-space and $\mathbb{Z}$ denotes the integers.) An $n$-gm $X, n \geq 5$, has the disjoint disks property $(D D P)$ if every pair of maps of the 2-cell $B^{2}$ into $X$ can be approximated arbitrarily closely by maps that have disjoint images. A subset $A$ of $X$ is $1-L C C$ in $X$ if for each $x \in A$ and neighborhood $U$ of $x$ in $X$, there is a neighborhood $V$ of $x$ in $X$ lying in $U$ such that the inclusion induced homomorphism $\pi_{1}(V \backslash A) \rightarrow \pi_{1}(U \backslash A)$ is trivial. A polyhedron $P$ in a (topological) manifold or polyhedron $M$ is tame in $M$, if, for every point $x \in P$, there is a triangulation of $P$ containing $x$ as a vertex and a triangulation of a neighborhood of $x$ in $M$ containing the star of $x$ in $P$ as a subcomplex. A subset $A$ of a (topological) manifold or a polyhedron $M$ is tame if it can be moved off of any tame 2-complex $Q$ in $M$ with an arbitrarily small isotopy of $M$ supported in a neighborhood of $A \cap Q$. In Štan'ko's terminology [17], the demension of $A$ is the same as $\operatorname{dim} A$. As a consequence, $A$ can be moved off of any tame polyhedron $P$, provided $\operatorname{dim} P+\operatorname{dim} A<\operatorname{dim} M$. (See [1], [17], and, especially [7] for an excellent discussion of "demension theory".) A codimension $\geq 3$ restriction is implied in this definition when $M$ is a manifold. If $M$ is a polyhedron, a codimension $\geq 3$ restriction on each essential stratum of $M$ is implied. If $M$ is a manifold and $\operatorname{dim} M \geq 5$, then tameness of $A \subset M$ is equivalent to the embedding $A$ being 1-LCC in $M$ $[1,18]$. We will want to extend this notion to certain codimension 2 subsets of a manifold.

Suppose $M$ is a (topological or $P L) m$-manifold, and that $A$ is an $(m-3)$ dimensional compactum. An embedding of $A \times I \subset M$ is tame if there is a collection $\left\{A_{1}, A_{2}, \ldots, A_{k}\right\}$ of compact subsets of $A$ whose interiors cover $A$, and a covering $\mathcal{U}=\left\{U_{i}\right\}_{i=1}^{k}$ of $A$ by a family of locally flat $m$-cells such that $\left(A_{i} \times I, A_{i} \times\{0,1\}\right) \subset$ 
$\left(U_{i}, \partial U_{i}\right), 1 \leq i \leq k$, and $\left(U_{i}, A_{i} \times I\right) \cong\left(I^{m-1}, A_{i} \times\{0\}\right) \times I$. If $g: A \rightarrow B$ is a map with mapping cylinder $C$, then an embedding $C \subset M$ is tame if $A \times[0, t]$ is tame in $M$ for all $t<1$.

If $f: A \rightarrow B$ is a map, then we set $S_{f}=\left\{x \in A: f^{-1} f(x) \neq x\right\}$. This is not the usual definition of the singular set of a map, one normally would define it as the closure of the set $S_{f}$ defined above, but it will serve us better in this paper.

Although the results of this section are stated for mappings between compacta, they are easily extended to locally compact spaces provided the maps are proper (that is, inverse images of compact sets are compact).

Proposition 2.2. Suppose that $X$ and $Y$ are compact $A N R$ 's and that $f: Y \rightarrow X$ is a $(k+1)$-connected map, $k \geq 0$. Suppose that $A$ is a closed, $k$-dimensional subset of $Y$ such that $B=f(A)$ has dimension $\leq k, C$ is the mapping cylinder of $f \mid A: A \rightarrow B$ with mapping cylinder projection $\gamma: C \rightarrow B$, and $D$ is the reduced mapping cylinder of $\gamma(\bmod A)$ with projection $\zeta: D \rightarrow B$. Then there is a map $g: C \rightarrow Y$ that extends the inclusion of $A$ into $Y$ and a map $h: D \rightarrow X$ that extends $f \circ g$ on $C$.

Moreover, given $\epsilon>0$ and $\mu>0$, there exists $\delta>0$ such that if $Z$ is a closed set in $B$ such that diam $f^{-1}(z)<\delta$ for all $z \in Z$, then we can choose $g$ and $h$ so that $\operatorname{diam} g\left(\gamma^{-1}(z)\right)<\mu$ and $\operatorname{diam} h\left(\zeta^{-1}(z)\right)<\epsilon$ for all $z \in Z$.

Proof. If $A$ and $B$ were polyhedra, then this would just be Stallings' argument in [16]. In order to handle arbitrary compacta, we approximate them by polyhedra and proceed as in [16].

Embed $Y$ and $X$ in some euclidean space $\mathbb{R}^{n}, n \geq 2 k+1$, and choose open sets $V \supset Y$ and $U \supset X$ along with retractions $s: V \rightarrow Y$ and $r: U \rightarrow X$. For any $\eta>0$ there is an $\eta$-map $\psi: B \rightarrow Q$, of $B$ to a polyhedron $Q$, of dimension $\leq k$, lying in a small neighborhood of $B$ in $U$. Extend $\psi \circ f \mid A$ to a mapping $\hat{f}: N \rightarrow Q$, where $N$ is a neighborhood of $A$ in $U$. For any given $\xi>0$ there is a $\xi$-map $\phi: A \rightarrow P$, of $A$ to a $k$-dimensional polyhedron $P$ in $N$. If $\xi$ is sufficiently small, the composition $\hat{f} \circ \phi: A \rightarrow Q$ will approximate $\psi \circ f \mid A$ closely enough so that there will be a small homotopy between them. Thus, we have the following diagram, where the right square is commutative and the left triangle is (small) homotopy commutative:

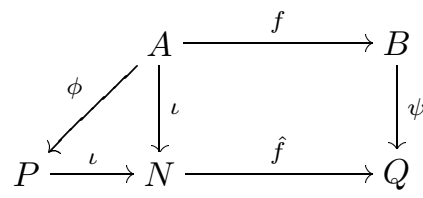

Set $\bar{f}=\hat{f} \mid P: P \rightarrow Q$. Let $\bar{C}$ be the mapping cylinder of $\bar{f}: P \rightarrow Q$ with projection $\bar{\gamma}: \bar{C} \rightarrow Q$. Let $\bar{D}$ be the reduced mapping cylinder of $\bar{\gamma}: \bar{C} \rightarrow Q(\bmod$ $P$ ) with projection $\bar{\zeta}: \bar{D} \rightarrow Q$. Then $\operatorname{dim} \bar{C} \leq k+1$, and $r \circ \bar{\gamma}: \bar{C} \rightarrow X$ is a map whose restriction to $P$ is homotopic to $f \circ s \mid P$. The Homotopy Extension Theorem provides a map $\alpha:(\bar{C}, P) \rightarrow\left(C_{f}, Y\right)$, extending $s$ on $P$ and $r$ on $Q$. Since $f$ is $(k+1)$-connected, $\alpha$ is homotopic, rel $\alpha \mid P$, to a map $\bar{g}: \bar{C} \rightarrow Y$. The homotopy provides a map of $\bar{C} \times I$ to $C_{f}$ whose composition with $\bar{\gamma}$ induces a map $\bar{h}: \bar{D} \rightarrow X$ extending $f \circ \bar{g}$ on $\bar{C}$. Let $E$ be the mapping cylinder of $\phi: A \rightarrow P$ and $F$ the reduced mapping cylinder of its projection $(\bmod A)$. Let $C^{\prime}$ be the mapping cylinder of $\psi \circ f: A \rightarrow Q$ with projection $\gamma^{\prime}$ and let $D^{\prime}$ be the mapping cylinder of $\gamma^{\prime}$ 
with projection $\zeta^{\prime}$. There are obvious maps $C \rightarrow C^{\prime}$ and $D \rightarrow D^{\prime}$. The Homotopy Extension Theorem gives a map (a homotopy equivalence) $C^{\prime} \rightarrow E \cup_{P} \bar{C}$ fixing $A$. Again, if $\xi$ is sufficiently small, there is a map $E \rightarrow Y$ extending the inclusion on $A$ and the retraction $s$ on $P$, and, thus, a map $F \cup_{P} \bar{D} \rightarrow X$ extending the inclusion on $A$. The map $g$ is then the composition

$$
C \rightarrow C^{\prime} \rightarrow E \cup_{P} \bar{C} \rightarrow Y .
$$

The map $h$ is a similar composition

$$
D \rightarrow D^{\prime} \rightarrow F \cup_{P} \bar{D} \rightarrow X .
$$

To see the "moreover" part, start with contractibility functions $T$ for $Y$ and $T^{\prime}$ for $X, \mu>0$, and $\epsilon>0$. Get sequences $0<\delta=\mu_{0}<\mu_{1}<\cdots<\mu_{k}=\mu$ and $0<\epsilon_{1}<\cdots<\epsilon_{k}<\epsilon_{k+1}=\epsilon$ such that $T\left(\mu_{i-1}\right)<\mu_{i}, T^{\prime}\left(\epsilon_{i}\right)<\epsilon_{i+1}$, and $f$ takes sets of diameter $<\mu_{i}$ to sets of diameter $<\epsilon_{i}, i=1,2, \ldots, k$. Choose a neighborhood $W$ of $Z$ in $B$ such that $f^{-1}(x)<\delta$ for $z \in W$, and choose $\eta$ sufficiently small so that, for some subpolyhedron $Q_{0} \subset Q, Z \subset \psi^{-1}\left(Q_{0}\right) \subset W$. We may also choose $\eta$ so that $\operatorname{diam}(\psi \circ f)^{-1}(z)<\delta$ for all $z \in Q_{0}$. We then choose $\xi$ small enough so that $\psi\left(\bar{f}^{-1}(z)\right)$ has diameter $<\delta$ for all $z \in Q_{0}$. During the inductive process in which we map $\bar{C}$, the mapping cylinder of $\bar{f}$, into $Y$, and $\bar{D}$ the reduced mapping cylinder of $\bar{\gamma}$ into $X$, use the local contractibility functions on $Y$ and $X$, respectively, instead of the connectivity of $f$ to map the submapping cylinder of $\bar{C}$ over $Q_{0}$ into $Y$ and the corresponding submapping cylinder $\bar{D}$ into $X$. We can make the image of the fibers of the mapping cylinder $E$ in $Y$ arbitrarily small, so that the image of the composite fibers over $Q_{0}$ of the composite $E \cup_{P} \bar{C}$ can be made $<\delta$. Finally, these fibers are approximations of the fibers of $C$ over $Q_{0}$. We deal with the fibers of $D$ over $Q_{0}$ in $X$ similarly.

The following statement is a corollary to the proof of Proposition 2.2.

Corollary 2.3. Suppose that $X$ and $Y$ are compact ENR's and $f: Y \rightarrow X$ is a map. For every $\epsilon>0$ there is a $\delta>0$ such that if $A \subset Y$ is compact, $B=f(A), C$ is the mapping cylinder of $f \mid A: A \rightarrow B$ with projection $\gamma: C \rightarrow B, D$ is the reduced mapping cylinder of $\gamma(\bmod A)$ with projection $\zeta: D \rightarrow B$, and $\operatorname{diam}\left(f^{-1}(x) \cap A\right)<$ $\delta$ for all $x \in B$, then there is a map $g: C \rightarrow Y$, extending the inclusion on $A$, and a map $h: D \rightarrow X$, extending $f \circ g$ on $C$ such that $\operatorname{diam} g \gamma^{-1}(x)<\epsilon$ and $\operatorname{diam} h \zeta^{-1}(x)<\epsilon$ for all $x \in B$.

The following notation will be used in Proposition 2.4 and Corollary 2.5:

$f: Y \rightarrow X$ is a mapping between compact ENR's,

$Z_{n}=\left\{x \in X: \operatorname{diam} f^{-1}(x) \geq 1 / n\right\}, n=1,2, \ldots$,

$Z=\bigcup_{n>1} Z_{n}$

$S_{n}=f^{-1}\left(Z_{n}\right)$,

$S=f^{-1}(Z)$, the singular set of $f$,

$C$ is the mapping cylinder of $f \mid S: S \rightarrow Z$ with projection $\gamma: C \rightarrow Z$,

$C_{n}=\gamma^{-1}\left(Z_{n}\right)$,

$D$ is the reduced mapping cylinder of $\gamma: C \rightarrow Z$ with projection $\zeta: D \rightarrow Z$, and

$D_{n}=\zeta^{-1}\left(Z_{n}\right)$.

Proposition 2.4. Suppose that $f$ is $(k+1)$-connected, $\operatorname{dim} S \leq k$, and $\operatorname{dim} Z \leq k$. Then there are functions $F: C \rightarrow Y$ and $H: D \rightarrow X$ such that

(1) F extends the inclusion on $S$, 
(2) $H$ extends $f \circ F$ on $C$,

(3) $F \mid C_{n}$ and $H \mid D_{n}$ are continuous for all $n \geq 1$,

(4) $\lim _{n \rightarrow \infty} \max \left\{\operatorname{diam} F\left(\gamma^{-1}(z)\right): z \in Z \backslash Z_{n}\right\}=0$, and

(5) $\lim _{n \rightarrow \infty} \max \left\{\operatorname{diam} H\left(\zeta^{-1}(z)\right): z \in Z \backslash Z_{n}\right\}=0$.

Proof. Condition (3) is equivalent to having $F$ and $H$ continuous in the weak topology generated by the compact subsets of $C$ and $D$, respectively. For a given $\epsilon_{0}>0$ and $\mu_{0}>0$, Proposition 2.2 implies that there exist $n_{0} \geq 1$ and mappings $g_{0}: S_{n_{0}} \rightarrow Y$, extending the inclusion on $S_{n_{0}}$, and and $h_{0}: D_{n_{0}} \rightarrow X$, extending $f \circ g_{0}$ on $D_{n_{0}}$, such that $\operatorname{diam} g_{0}\left(\gamma^{-1}(z)\right)<\mu_{0}$ and $\operatorname{diam} h_{0}\left(\zeta^{-1}(z)\right)<\epsilon_{0}$ for $z \in\left(Z_{n_{0}} \cap \mathcal{C} \ell\left(Z_{n_{0}+1} \backslash Z_{n_{0}}\right)\right)$. Set $W_{i}=\mathcal{C} \ell\left(Z_{n_{0}+i} \backslash Z_{n_{0}+i-1}\right), i=1,2, \ldots$, and $W_{0}=Z_{n_{0}}$. Apply Proposition 2.2 to get $g_{i}: \gamma^{-1}\left(W_{i}\right) \rightarrow Y$, extending the inclusion on $f^{-1}\left(W_{i}\right)$, and $h_{i}: \zeta^{-1}\left(W_{i}\right) \rightarrow X$, extending $f \circ g_{i}$ on $\gamma^{-1}\left(W_{i}\right)$, so that $\max \left\{\operatorname{diam} g_{i}\left(\gamma^{-1}(z)\right): z \in W_{i}\right\}=\mu_{i}$ and $\max \left\{\operatorname{diam} h_{i}\left(\zeta^{-1}(z)\right): z \in W_{i}\right\}=\epsilon_{i}$, where $\mu_{i}, \epsilon_{i} \rightarrow 0$ as $i \rightarrow \infty$. We can assume that $\mu_{0}, \mu_{1}, \ldots$ and $\epsilon_{0}, \epsilon_{1}, \ldots$ are sufficiently small so that $g_{i} \mid \gamma^{-1}\left(W_{i-1} \cap W_{i}\right)$ is $\mu_{i-1}$-homotopic to $g_{i-1} \mid \gamma^{-1}\left(W_{i-1} \cap W_{i}\right)$ in $Y$, rel $f^{-1}\left(W_{i-1} \cap W_{i}\right)$, and $h_{i} \mid \zeta^{-1}\left(W_{i-1} \cap W_{i}\right)$ is $\epsilon_{i-1}$-homotopic to $h_{i-1} \mid \zeta^{-1}\left(W_{i-1} \cap\right.$ $\left.W_{i}\right)$ in $X$, rel $f \circ g_{i} \mid \gamma^{-1}\left(W_{i-1} \cap W_{i}\right)$. The Homotopy Extension Theorem allows us to get $g_{i} \mu_{i-1}$-homotopic to $g_{i}^{\prime}$ so that $g_{i}^{\prime}=g_{i-1}$ on $\gamma^{-1}\left(W_{i-1} \cap W_{i}\right)$ and $g_{i}^{\prime}=g_{i}$ on $\gamma^{-1}\left(W_{i} \cap W_{i+1}\right)$ (and $\left.g_{0}^{\prime}=g_{0}\right)$. Piecing the $g_{i}^{\prime}$ 's together gives the map $F$. A similar process yields $H$.

Corollary 2.5. Given $\epsilon>0$ there exists $\delta>0$ such that if $\operatorname{diam} f^{-1}(x)<\delta$ for all $x \in Z$, then there are functions $F: C \rightarrow Y$ and $H: D \rightarrow X$ such that

(1) $F$ extends the inclusion on $S$ and $H$ extends $f \circ F$ on $C$,

(2) $F \mid C_{n}$ and $H \mid D_{n}$ are continuous for all $n \geq 1$,

(3) $\operatorname{diam} F\left(\gamma^{-1}(z)\right)<\epsilon$ and $\operatorname{diam} H\left(\zeta^{-1}(z)\right)<\epsilon$ for all $z \in Z$, and

(4) $\lim _{n \rightarrow \infty} \max \left\{\operatorname{diam} F\left(\gamma^{-1}(z)\right): z \in Z \backslash Z_{n}\right\}=0$, and

(5) $\lim _{n \rightarrow \infty} \max \left\{\operatorname{diam} H\left(\zeta^{-1}(z)\right): z \in Z \backslash Z_{n}\right\}=0$.

Definition 2.6. By a pseudo-isotopy of a space $M$, we mean a homotopy $H_{t}: M \rightarrow$ $M, t \in I$, such that $H_{t}$ is a homeomorphism for $0 \leq t<1$.

Observe that the mapping cylinder $C$ of a map $f: A \rightarrow B$ has a natural "linear" homotopy $h_{t}: C \rightarrow C, t \in I$, with $h_{0}=\mathrm{id}$, that shrinks each mapping cylinder fiber $\gamma^{-1}(y)$ to $y, y \in B$.

Lemma 2.7. Suppose that $f: A \rightarrow B$ is a mapping between compact spaces, with mapping cylinder $C$ and mapping cylinder projection $\gamma: C \rightarrow B$, and suppose that $C \subset M$ is a tame embedding of $C$ into a manifold $M, \operatorname{dim} M-\operatorname{dim} A \geq 3$. Given $\epsilon>0$ and a neighborhood $U$ of $C \backslash B$, there is a pseudo-isotopy $H_{t}$ of $M, t \in I$, such that

(1) $H_{0}=\mathrm{id}$,

(2) $H_{t}=$ id on $M \backslash U$ for all $t \in I$,

(3) $H_{t} \mid C$ is the natural shrinking homotopy of $C$ to $B$,

(4) for all $x \in M$, either $H_{t}(x)=x$ for all $t \in I$ or there exists $a \in A$ such that $H(x, t) \in N_{\epsilon}(a \times I)$, and

(5) $H_{1}$ is 1-1 on $M \backslash C$.

We shall call the pseudo-isotopy $H_{t}$ of Lemma 2.7 an $\epsilon$-shrinking of $C$ to $B$ in $U$. We will use this lemma to shrink certain upper semicontinuous decompositions 
of a manifold $M$. Recall that a decomposition of a space $M$ into compacta $\mathcal{C}$ is upper semicontinuous (usc) if for every $\gamma \in \mathcal{C}$ and every neighborhood $U$ of $\gamma$ there is a neighborhood $V$ of $\gamma$ such that if $\gamma^{\prime} \in \mathcal{C}$ and $\gamma^{\prime} \cap V \neq \varnothing$, then $\gamma \subset U$. A usc decomposition $\mathcal{C}$ of a space $M$, with quotient map $p: M \rightarrow M / \mathcal{C}$, is shrinkable if for every $\epsilon>0$ there is an isotopy $h_{t}, t \in I$, such that $\operatorname{diam} h_{1}(\gamma)<\epsilon$ for all $\gamma \in \mathcal{C}$ and $d\left(p, p \circ h_{t}\right)<\epsilon$ for all $t \in I$. The Bing Shrinking Criterion states that if $\mathcal{C}$ is a shrinkable usc decomposition of $M$, then $M / \mathcal{C} \cong M$ and there are pseudo-isotopies $h_{t}, t \in I$, with arbitrarily small tracks in $M / \mathcal{C}$, shrinking precisely the elements of $\mathcal{C}$ to points. (See $[6]$.)

There is a homotopy version of Lemma 2.7 for 1-LCC embeddings of mapping cylinders in a generalized manifold.

Lemma 2.8. Suppose that $f: A \rightarrow B$ is a mapping between compact spaces, with mapping cylinder $C$ and mapping cylinder projection $\gamma: C \rightarrow B$, and suppose that $C \subset M \subset X$, where $M$ is an ANR, 1-LCC embedded in an $n$-gm $X, n \geq 5$. Given $\epsilon>0$ and a neighborhood $U$ of $C \backslash B$, there is a homotopy $H_{t}, t \in I$, of $X$ such that

(1) $H_{0}=\mathrm{id}$,

(2) $H_{t}=$ id on $X \backslash U$ for all $t \in I$,

(3) $H_{t} \mid C$ is the natural shrinking homotopy of $C$ to $B$,

(4) $H_{t}(X \backslash M) \subset X \backslash M$ for all $t \in I$, and

(5) for all $x \in X$, either $H_{t}(x)=x$ for all $t \in I$ or there exists $a \in A$ such that $H(x, t) \in N_{\epsilon}(a \times I)$.

Proof. The Homotopy Extension Theorem provides a homotopy $H_{t}, t \in I$, of $X$ having all of the above properties except (4). Property (4) is obtained by applying Corollary 2.7 of [15]. In the terminology of [15], $H_{t}$ is a strict homotopy of the pair $(X, M)$.

We will call the homotopy $H_{t}, t \in I$, of $X$ a strict homotopy $\epsilon$-shrinking of $C$ to $B$ in $U$ rel $M$.

We will want to apply the shrinking lemmas to shrink $C$ to $B$ "a little at a time". To set this up, we will need some additional notation.

Given $g: C \rightarrow X$ and $\epsilon>0$, define an $\epsilon$-layering of $C$ (relative to $g$ ) to be a sequence of maps $\alpha_{0}, \ldots, \alpha_{\ell}: A \rightarrow I$ such that

(a) $\alpha_{0}(x)=1 \geq \alpha_{1}(x) \geq \ldots \geq \alpha_{\ell}(x)=0$ for all $x \in A$,

(b) $\alpha_{1}(x)=0$ if $x \in g^{-1}\left(B_{\epsilon / 2}\right)$,

(c) $\operatorname{diam} g\left(x \times\left[\alpha_{j}(x), \alpha_{j-1}(x)\right]\right)<\epsilon$ for all $x \in A$.

For a given layering of $C$, call $C[j]=\left\{\left(x, \alpha_{j}(x)\right): x \in A\right\}$ the $j$ th slice, $0 \leq j \leq \ell$, and $C[j-1, j]=\left\{(x, t) \in A \times I: \alpha_{j}(x) \leq t \leq \alpha_{j-1}(x)\right\}$ the $j$ th layer, $1 \leq j \leq \ell$. (Observe that $C[0]=B$ and $C[\ell]=A$.) (See Figure 2.1.) Thus there is a natural $\epsilon$-homotopy of $g \mid C[j]$ to $g \mid C[j-1]$ with support in $C[j-1, j]$. With the help of Urysohn functions one can easily show that $\epsilon$-layerings exists for any $\epsilon>0$.

We conclude this section with a statement of the main result of [2] and two of its consequences that will be useful in the present work. (See also [20].)

Theorem 2.9 ([2]). Suppose that $X$ an $n$-gm with the DDP, $n \geq 5, P$ and $Q$ are polyhedra of dimensions $p$ and $q$, respectively, and $f: P \rightarrow X$ and $g: Q \rightarrow X$ are maps. The $f$ and $g$ can be approximated by maps $f^{\prime}$ and $g^{\prime}$ such that 


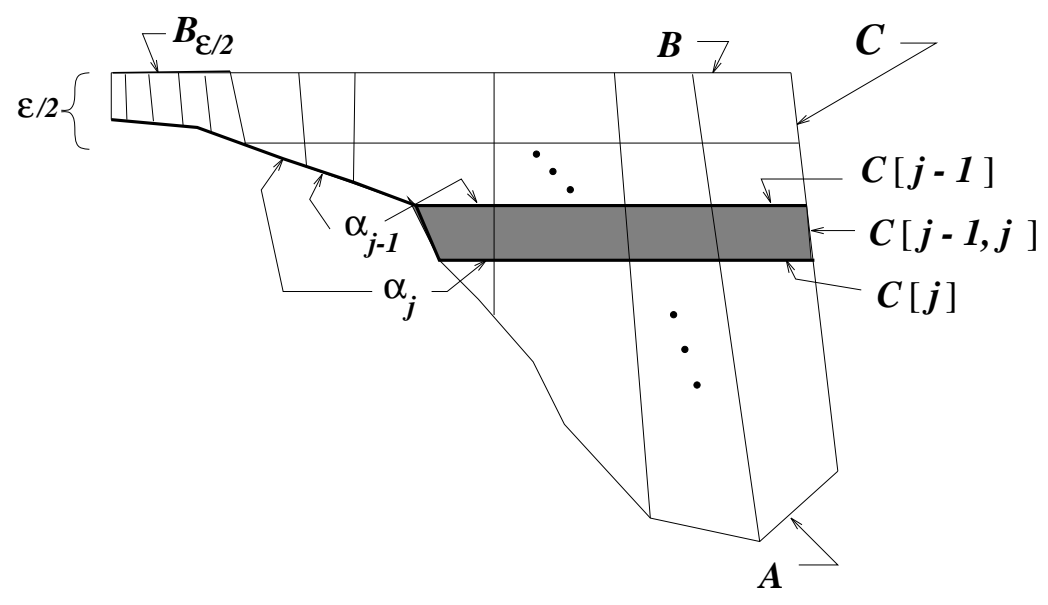

FiguRE 2.1

(1) $\operatorname{dim}\left[f^{\prime}(P) \cap g^{\prime}(Q)\right] \leq p+q-n$,

(2) $f^{\prime}(P)$ and/or $g^{\prime}(Q)$ is 1-LCC in $X$ if $n-p \geq 3$ and/or $n-q \geq 3$, and

(3) $f^{\prime}(P) \cap g^{\prime}(Q)$ is $1-L C C$ in $X$ if $p+q-n \leq n-3$.

Implicit in the proof of Theorem 2.9 in [2] is the fact that if, say, $\operatorname{dim} f(P) \leq m-3$ and $f(P)$ is already 1-LCC in $X$, then we can let $f^{\prime}=f$.

Corollary 2.10. Suppose that $f: P \rightarrow X$ is a map of a p-dimensional complex into an $n$-gm $X$ with the $D D P, n-p \geq 3$. Then $f$ can be approximated by $f^{\prime}: P \rightarrow X$ such that

(1) $f^{\prime}(P)$ is a 1-LCC subset of $X$ of dimension $\leq p$,

(2) $\operatorname{dim} S_{f^{\prime}} \leq 2 p-n$,

(3) each compact subset of $S_{f^{\prime}}$ is a tame subset of $P$, and

(4) $f^{\prime}\left(S_{f^{\prime}}\right)$ is a 1-LCC subset of $X$ of dimension $\leq 2 p-n$.

Proof. Let $T_{1}, T_{2}, \ldots$ be a sequence of subdivisions of some triangulation of $P$ with mesh $\rightarrow 0$. Apply Theorem 2.9 inductively to the skeleta of $T_{i}$ to get a map $f^{\prime}: P \rightarrow X$ such that $S_{f^{\prime}} \subset \bigcup_{i}\left(P \backslash T_{i}^{(n-p-1)}\right)$, where $T_{i}^{(k)}$ denotes the $k$-skeleton of $T_{i}$. Classical results of [10] show that $\operatorname{dim} \bigcup_{i}\left(P \backslash T_{i}^{(n-p-1)}\right) \leq 2 p-n$.

Corollary 2.11. Suppose that $f: P \rightarrow X$ is a map of a p-dimensional compactum into an $n$-gm $X$ with the $D D P, n-p \geq 3$. Then $f$ can be approximated by $f^{\prime}: P \rightarrow$ $X$ such that

(1) $f^{\prime}(P)$ is a 1-LCC subset of $X$ of dimension $\leq p$,

(2) $\operatorname{dim} S_{f^{\prime}} \leq 2 p-n$, and

(3) $f^{\prime}\left(S_{f^{\prime}}\right)$ is a 1-LCC subset of $X$ of dimension $\leq 2 p-n$.

Proof. Apply Corollary 2.10 to an inverse system of polyhedra converging to $P$.

\section{A special CASE}

In this section, we apply the shrinking lemmas of section 2 to describe our main geometric construction. The arguments will demonstrate that, in this range of dimensions, a Whitney Trick can be made to work for mappings into a generalized 
manifold with the $D D P$ without the extra complications arising when mapping cylinders over singularities do not embed by virtue of general position alone.

The Main Construction. Let $X^{n}, n \geq 5$, be a compact $n$-gm with the DDP and $M$ a closed $P L m$-manifold, $3 m \leq 2 n-3$. Given a $(2 m-n+1)$-connected map $f: M \rightarrow X$, we construct a homotopy of $f$ to a $1-L C C$ embedding $f^{\prime}: M \rightarrow X$.

By Corollary 2.10 we may assume that $S_{f}$ is a tame subset of $M$. Set $S=S_{f}$. Let $Z=f(S)$, let $C$ be the mapping cylinder of $f \mid S: S \rightarrow Z$ with projection $\gamma: C \rightarrow Z$, and let $D$ be the reduced mapping cylinder of $\gamma: C \rightarrow Z(\bmod S)$ with projection $\zeta: D \rightarrow Z$. For $r>0$, set $Z_{r}=\left\{z \in Z: \operatorname{diam} f^{-1}(z) \geq r\right\}, S_{r}=f^{-1}\left(Z_{r}\right)$, and $C_{r}=\gamma^{-1}\left(Z_{r}\right)$. Apply Proposition 2.4 to get functions $F: C \rightarrow M$ and $\bar{F}: D \rightarrow X$ such that

(i) $F$ extends the inclusion on $S$ and $\bar{F}$ extends $f \circ F$ on $C_{r}$,

(ii) $F \mid C_{r}$ and $\bar{F} \mid D_{r}$ are continuous for all $r>0$,

(iii) $\lim _{r \rightarrow 0} \max \left\{\operatorname{diam} F\left(\gamma^{-1}(z)\right): z \in Z \backslash Z_{r}\right\}=0$, and

(iv) $\lim _{r \rightarrow 0} \max \left\{\operatorname{diam} \bar{F}\left(\zeta^{-1}(z)\right): z \in Z \backslash Z_{r}\right\}=0$.

We may assume $m \geq 3$ (and $n \geq 6$ ); otherwise, the general position results of [2] apply directly. Since $2(k+1)+1 \leq m$, we may also assume that $F \mid C_{r}$ is a tame embedding for each $r>0$. By Corollary 2.11 we may assume that $\bar{F} \mid D_{r}$ is a 1-LCC embedding for each $r>0$.

Assertion 3.1. The collection $\mathcal{C}$ of subsets of $M$ consisting of $\left\{F\left(\gamma^{-1}(z)\right): z \in Z\right\}$ together with the remaining points of $M$ form a shrinkable, usc decomposition of $M$. In particular, for every $\epsilon>0$, there is a pseudo-isotopy $h_{t}: M \rightarrow M, t \in I$, such that $\mathcal{C}=\left\{h_{1}^{-1}(x): x \in M\right\}$ and $d\left(p, p h_{t}\right)<\epsilon$, where $p: M \rightarrow M / \mathcal{C}$ is the quotient map.

Proof. In order to simplify the notation, we shall drop the $F$ and identify $C$ with $F(C)$ (although $F$ most likely will not be a homeomorphism!).

First, the fact that $\mathcal{C}$ is usc follows easily from properties of $F$ and the fact that $\left\{f^{-1}(x): x \in f(M)\right\}$ is usc.

Let $p: M \rightarrow M / \mathcal{C}$ be the quotient map. Given $\epsilon>0$, we will find an $i \geq 1$, with $\delta=1 / i<\epsilon$, and an ambient isotopy $H_{t}: M \rightarrow M, 0 \leq t \leq 1$, such that

(a) $H_{0}=$ id,

(b) $H_{t}=$ id on $M \backslash N_{\delta}\left(C_{i}\right)$,

(c) $d\left(p \circ H_{t}, p\right)<\epsilon$

(d) $\operatorname{diam} H_{1}\left(\gamma^{-1}(z)\right)<\epsilon$, for all $z \in Z$.

Fix a $\delta>0$ and let $\alpha_{0}, \ldots, \alpha_{\ell}$ be a $\delta$-layering of the mapping cylinder $C_{\delta / 2}$ (relative to $F$ ) with slices $C[j], 0 \leq j \leq \ell$, and layers $C[j-1, j], 1 \leq j \leq \ell$.

Choose a neighborhood $U_{1}$ of $C[\ell-1, \ell]$, rel $C[\ell-1]$, such that if $z \in Z \backslash Z_{\delta / 2}$ and $\gamma^{-1}(z) \cap U_{1} \neq \varnothing$, then $\operatorname{diam} \gamma^{-1}(z)<\delta$. For $\mu_{1}>0$, to be determined later, apply Lemma 2.7 to get a $\mu_{1}$-shrinking $H_{t}^{1}, t \in I$, of $C[\ell-1, \ell]$ to $C[\ell-1]$ in $U_{1}$.

Inductively, for $2 \leq j \leq \ell$, choose a neighborhood $U_{j}$ of $C[\ell-j, \ell-j+1]$, rel $C[\ell-j]$, such that if $z \in Z \backslash Z_{\delta / 2}$ and $H_{1}^{j-1} \circ \cdots \circ H_{1}^{1}\left(\gamma^{-1}(z)\right) \cap U_{j} \neq \varnothing$, then $\operatorname{diam}\left(H_{1}^{j-1} \circ \cdots \circ H_{1}^{1}\left(\gamma^{-1}(z)\right)\right)<\delta$. For $\mu_{j}>0$, to be determined later, apply Lemma 2.7 to get a $\delta$-shrinking $H_{t}^{j}, t \in I$, of $C[\ell-j, \ell-j+1]$ to $C[\ell-j]$.

If $\delta<\epsilon / 3$, then $\operatorname{diam}\left(H_{1}^{\ell} \circ \cdots \circ H_{1}^{1}\left(\gamma^{-1}(z)\right)\right)<\epsilon$ for all $z \in Z$. If $\mu_{1}, \ldots, \mu_{\ell}$ are chosen correctly, then the composition of the pseudo-isotopies $H^{1}, \ldots, H^{\ell}$ (as 
homotopies) of $M$ is approximable by a pseudo-isotopy of $M$ with the desired properties.

We now wish to perform a shrinking in $X$ that will provide the improvement on the connectivity of $f$. The collection $\mathcal{D}=\left\{\zeta^{-1}(z), z \in Z\right\}$, together with the remaining points of $X$, forms a usc decomposition of $X$. Although we don't know, at least at this time, how to isotopically shrink this decomposition of $X$, Lemma 2.8 will provide a homotopy of $f$ to the desired $f^{\prime}$ at the expense of introducing new singularities, whose sizes we are able to control.

Assertion 3.2. Let $h: M \rightarrow M$ be the map as above. For every $\epsilon>0$ and $\mu>0$, there is a homotopy $G_{t}, t \in I$, of $X, \epsilon$-small over $X / \mathcal{D}$, such that $G_{0}=\mathrm{id}$, $G_{1}: X \rightarrow X$ shrinks the elements of $\mathcal{D}$ to points, and, if $g=G_{1} \circ f \circ h^{-1}: M \rightarrow X$ is the induced map, then $\operatorname{diam} g^{-1}(x)<\mu$ for all $x \in g(M)$.

Proof. Observe that the reduced mapping cylinder $D$ of $\gamma: C \rightarrow Z(\bmod S)$ is, in a natural way, (fiber-wise) homeomorphic to the unreduced mapping cylinder $C_{\gamma}$ of $\gamma$. There is also another copy of $C$ in $C_{\gamma}$, namely, the submapping cylinder of $C_{\gamma}$ over $S$. The union of these two copies of $C$ forms the image of $C$ in the quotient of $C_{\gamma}$ that gives $D$. We wish to use the mapping cylinder parameter of the unreduced mapping cylinder to shrink the fibers of $D$. Drop the $\bar{F}$ and identify $C_{\gamma}$ with $\bar{F}\left(C_{\gamma}\right)$ as before.

Recall that $C_{\gamma}=C \times I / \sim$ where $(c, 1) \sim \gamma(c)$. Fix $\delta>0$, and set

$$
\begin{aligned}
Z_{\delta / 2} & =\left\{z \in Z: \zeta^{-1}(z) \geq \delta / 2\right\}, \\
D_{\delta / 2} & =\zeta^{-1}\left(Z_{\delta / 2}\right), \text { and } \\
C_{\delta / 2} & =\gamma^{-1}\left(Z_{\delta / 2}\right) .
\end{aligned}
$$

Let $\alpha_{0}, \ldots, \alpha_{\ell}$ be a $\delta$-layering of $D_{\delta / 2}($ relative to $\bar{F}$ ), with respect to the unreduced mapping cylinder structure, with slices $D[j], 0 \leq j \leq \ell$, and layers $D[j-1, j]$, $1 \leq j \leq \ell$.

Repeat the argument in Assertion 3.1 using Lemma 2.8. That is, for the mapping cylinder $D_{\delta / 2}$ of $\zeta: C_{\delta / 2} \rightarrow Z_{\delta / 2}$ and for $\mu_{j}>0,1 \leq j \leq \ell$, and neighborhood $U_{j}$ of $D[j-1, j]$ rel $D[j-1]$, get a homotopy $\mu_{j}$-shrinking $H_{t}^{j}, t \in I$, of $D[j-1, j]$ to $D[j-1]$ in $U_{j}$. (We needn't be very careful with the strictness of this homotopy; general position will keep critical sets separated.)

Each $H_{t}^{j}$ will introduce new singularities on $f$. Since the image of the singularities of $f$ meet $D$ at $D[0]=Z_{\delta}$, any new singularities introduced in $H_{t}^{j}$ can be made to have diameter $<\mu / 2$ in $M$ and, by general position, have images disjoint from $D$. The neighborhoods in which the shrinking takes place in $X$ are chosen small enough so that their point-inverses under $f$ are close enough to a fiber $\gamma^{-1}(z)$ of $C$ so that the shrinking isotopy $H_{t}$ of $M$ moves them into the $\mu / 2$-neighborhood of $H_{1}\left(\gamma^{-1}(z)\right)$.

Apply this process inductively over $Z_{1 / k}, k=1,2, \ldots$, introducing new singularities of diameter $<\mu / 2^{k}$ in $M$. In the limit we produce a homotopy of $X$ with the desired properties.

Apply Assertions 3.1 and 3.2 inductively to get a sequence of maps $\left\{f_{i}, i \geq 0\right\}$ of $M$ to $X$ with smaller and smaller point-inverses. Our assumptions guarantee that the homotopies from $f_{i-1}$ to $f_{i}$, from the second stage on, will be controlled not only over the quotient spaces, but over $M$ and $X$ as well. The Bing Shrinking 
Criterion will ensure that the sequence will converge to an embedding of $M$ in $X$ (homotopic to $f$ ). The 1-LCC condition is obtained by avoiding a dense set of embeddings of 1-LCC 2-cells in $X$ as in [2].

This concludes the Main Construction.

One easily establishes versions for manifolds with boundary. If $f \mid \partial M$ is an embedding, then the fibers of $C$ meet $\partial M$ in at most one point. Instead of working with the mapping cylinder $C$ over the singularities, use the reduced mapping cylin$\operatorname{der}\left(\bmod f^{-1}(Z) \cap \partial M\right)$. This allows us to shrink the decompositions of $M$ keeping $\partial M$ fixed. In $X$, reduce the mapping cylinder $D$ over $Z \cap f(\partial M)$. This allows us to homotopically shrink the decompositions of $X$ keeping $f(\partial M)$ fixed. If $f \mid \partial M$ is not an embedding, apply the process to $\partial M$ first to get $f$ to be an embedding on $\partial M$.

Theorem 3.3. Let $X^{n}, n \geq 5$, be a compact $n$-gm with the DDP, $(M, \partial M)$ a compact $P L$ m-manifold with boundary, $3 m \leq 2 n-3$, and $f: M \rightarrow X a(2 m-n+1)$ connected map such that $f \mid \partial M$ is $(2 m-n-1)$-connected. Then $f$ is homotopic to a 1-LCC embedding $f^{\prime}: M \rightarrow X$. Moreover, if $f \mid \partial M$ is an embedding, then we can get $f^{\prime}|\partial M=f| \partial M$.

The arguments presented also yield a controlled version of this result.

Theorem 3.4. Suppose that $X^{n}, n \geq 5$, is a generalized $n$-manifold with the DDP, and $(M, \partial M)$ is a compact $P L m$-manifold with boundary, $3 m \leq 2 n-3$. For every $\epsilon>0$ there exist $\delta>0$ and $\eta>0$ such that if $f: M \rightarrow X$ is $(\delta, 2 n-m+1)$-connected and $f \mid \partial M$ is $(\eta, 2 m-n-1)$-connected, then $f$ is $\epsilon$-homotopic to a 1-LCC embedding $f^{\prime}: M \rightarrow X$. Moreover, if $f \mid \partial M$ is an embedding, then we can get $f^{\prime}|\partial M=f| \partial M$.

\section{Proof of Theorems 1.1 And 1.2}

In order to steal the one dimension, from $3 m \leq 2 n-3$ to $3 m \leq 2 n-2$, we will have to deal with 0-dimensional singularities in the function $F: C \rightarrow M$ that maps the mapping cylinder over the singularities of $f$ into $M$ as well as 0 -dimensional intersections of $\bar{F}(D)$ with $f(M)$.

Lemma 4.1. Suppose that $A$ is a $(k-1)$-dimensional compactum and $g: A \times I \rightarrow$ $M$ is a mapping into a PL $2 k$-manifold $M$ such that $g \mid(A \times\{0,1\})$ is a tame embedding. Then for any $\epsilon>0$ there is a collection $A_{1}, A_{2}, \ldots, A_{m}$ of compact subsets of $A$ whose interiors cover $A$ and an $\epsilon$-homotopy rel $g \mid(A \times\{0,1\})$ of $g$ to an immersion $g^{\prime}: A \times I \rightarrow M$ such that $g^{\prime} \mid\left(A_{i} \times I\right)$ is a tame embedding for each $i=1,2, \ldots, m$.

Proof. Given $\mu>0$ it is not difficult to get a map $\phi: A \rightarrow P$, where $P$ is a $k$ dimensional polyhedron and a map $\bar{g}: P \times I \rightarrow M$ such that $g$ is $\mu$-homotopic to $\bar{g} \circ(\phi \times$ id $)$. Without loss of generality, $\bar{g}$ is an immersion whose singular set meets each $x \times I \subset P \times I$ in at most one point and whose restriction to each $P \times\{t\}$ is an embedding for all $t \in I$. Let $W$ be a regular neighborhood of $\bar{g}(P \times I)$ that pulls back to an abstract (2k-dimensional) regular neighborhood $\bar{W}$ of $P \times I$, and let $G: \bar{W} \rightarrow W$ be the associated immersion. There is an isotopy of $\bar{W} \cup$ (collar on $\partial W$ ) taking $P \times\{0\}$ into $\partial W$. Regular neighborhood theory then implies that $\bar{W} \cong N \times I$, where $N$ is an abstract $(2 k-1)$-dimensional regular neighborhood of $P$, and that a relative regular neighborhood $\bar{N}$ of $P \times I$, rel $P \times\{0,1\}$ satisfies 
$(\bar{N}, P \times I) \cong(N, P) \times I$. Thus, we have an immersion $G \mid N \times I: N \times I \rightarrow M$ extending $\bar{g}$. We may assume that $G \mid N \times\{t\}$ is an embedding for all $t \in I$.

The map $\bar{g} \circ \phi: A \rightarrow \bar{g}(P) \subset G(N \times\{0\})$ can be approximated by a tame embedding $\bar{\phi}: A \rightarrow G(N \times\{0\})$. Pull back $\bar{\phi}$ to a tame embedding $\psi: A \rightarrow$ $N(=N \times\{0\})$. Then $G^{\prime}=G \circ(\psi \times$ id $): A \times I \rightarrow M$ is an immersion whose singular set meets each $x \times I \subset A \times I$ in at most one point and whose restriction to each $A \times\{t\}$ is a tame embedding. By results of [1] and [17] $G^{\prime} \mid A \times\{0,1\}$ and $g \mid A \times\{0,1\}$ are $\mu$-ambient isotopic in $M$. (This applies even when $k=2$.) Thus $G^{\prime}$ is $\mu$-isotopic to an immersion $g^{\prime}: A \times I \rightarrow M$ satisfying singularity conditions similar to those on $G$. If $\mu$ is sufficiently small, then $g$ and $g^{\prime}$ are $\epsilon$-homotopic, rel $A \times\{0,1\}$. The conditions on the singularities of $g^{\prime}$ imply the existence of compact sets $A_{1}, A_{2}, \ldots, A_{m}$ in $A$ such that each $g^{\prime} \mid\left(A_{i} \times I\right)$ is tame.

Corollary 4.2. Suppose that $A$ is a $(k-1)$-dimensional compactum and $g: A \times I \rightarrow$ $M$ is a mapping into a PL $2 k$-manifold $M$ such that $g \mid(A \times\{0,1\})$ is a tame embedding. Let $g^{\prime}: A \times I \rightarrow M$ be an immersion close to $g$ as in the conclusion of Lemma 4.1. Then there is a finite set $a_{1}, \ldots, a_{n} \in A$ and a regular homotopy of $g^{\prime}$ rel $g^{\prime} \mid A \times\{0,1\}$, supported on a neighborhood of $g^{\prime}\left(a_{1} \times I\right) \cup \cdots \cup g^{\prime}\left(a_{n} \times I\right)$, to a tame embedding $g^{\prime \prime}: A \times I \rightarrow M$.

Proof. The immersion $\bar{g}: P \times I \rightarrow M$ of the polyhedron $P$ in the proof of Lemma 4.1 used to get the immersion of $A \times I$ has a finite number of double-point singularities of the form $(x, s),(y, t) \in P \times I$ with $\bar{g}(x, s)=\bar{g}(y, t), x \neq y, 0<s, t<1$, where $x$ and $y$ lie in the interiors of disjoint $k$-simplexes $\sigma$ and $\tau$, respectively, in $P \times I$. We can "pipe" $\bar{g} \mid \tau$ off of $\bar{g}(\sigma)$ along the $\operatorname{arc} \bar{g}(x \times[0, s])$ inside a $2 k$-cell neighborhood $B$ of $\bar{g}(x \times[0, s])$. The result is a regular homotopy of $\bar{g}$, supported in a disjoint union of $2 k$-cells to an embedding $\bar{g}^{\prime}: P \times I \rightarrow M$, hence, an embedding of $N \times I$ in $M$. The restriction of the regular homotopy to $A \times I$ gives the desired map $g^{\prime}$.

Proof of Theorems 1.1 and 1.2. The only case not covered above is $3 m=2 n-2$ and $m=2 k$ is even. Get $f: M \rightarrow X$ in general position as before so that compact subsets of $S=\left\{x \in M: f^{-1} f(x) \neq x\right\}$ are tame in $M, Z=f(S)$ is 1-LCC in $X$, and $\operatorname{dim} S, \operatorname{dim} Z \leq k$. If $C$ denotes the mapping cylinder of $f \mid S: S \rightarrow Z$, then we can use Corollary 4.2 to get a function $F: C \rightarrow M$, as in the proof of Theorem 3.3, except that $F$ is an immersion on compact submapping cylinders of $C$ and has no singularities mapping to $\partial M$. Use Corollary 4.2 to remove the singularities. Next, the mapping cylinder $D(=\bar{F}(D) \subseteq X)$ over $C$ may have 0-dimensional intersection with $f(M)$ disjoint from $f(C)$. Let $A$ denote this set. As a subset of $D \backslash Z, A$ projects to a subset $B_{1}$ of $Z$, via a mapping $\alpha_{1}=\zeta \mid A: A \rightarrow B_{1}$. By general position results of $[2,20], f: M \rightarrow X$ may be assumed to have no triple points; hence, the map $f \mid S: S \rightarrow Z$, restricted to any $S_{\epsilon}$, is a locally trivial fibration with fiber the 0 -sphere. Thus, since $A$ is 0 -dimensional, the map $\alpha_{1}: A \rightarrow Z$ lifts to a mapping $\alpha_{2}: A \rightarrow B_{2} \subseteq S$. The mapping cylinder $C_{1}$ of $\alpha_{1}$ naturally embeds in $D$ (in the weak topology). After identifying $A$ with $f^{-1}(A) \subseteq M$, we can apply connectivity assumptions to embed the mapping cylinder $C_{2}$ of $\alpha_{2}$ in $M$ so that $C_{2}$ is 1-LCC in $M$ and meets $C$ at $B_{2}$. By general position we may assume $f\left(C_{2}\right) \cap C_{1}=A \cup B_{1}$. Let $D^{\prime}$ be the mapping cylinder of the natural map $\beta: f\left(C_{2}\right) \cup C_{1} \rightarrow B_{1}$. Apply connectivity conditions and general position to assume that $D^{\prime}$ is 1-LCC embedded in $X$ so that $D^{\prime} \cap[f(M) \cup D]=f\left(C_{2}\right) \cup C_{1}$. The proof now continues as in the Main 
Construction with the obvious changes. That is, we isotopically shrink $C \cup C_{2}$ to the copy of $Z$ in $M$ and homotopically shrink $D \cup D^{\prime}$ to $Z$ in $X$.

\section{General position Revisited}

The general position results of [2] allow an adjustment of a map $f: M \rightarrow X$ so that the singular set $S_{f}=\left\{x \in M: f^{-1} f(x) \neq x\right\}$ has dimension $\leq 2 m-n$ (Corollary 2.10). In this section we show that, in the meta-stable range, any map of a $P L$ manifold $M$ into an $n$-gm can be adjusted so that its singularities have the same structure as if we were mapping $M$ to a $P L$ manifold. First, we establish a lemma showing how to reduce singularities to compacta of the expected dimension.

Lemma 5.1. Suppose that $X^{n}, n \geq 5$, is an $n$-gm with the DDP, $M^{m}$ is a compact $P L m$-manifold, $3 m \leq 2 n-2$, and $f: M \rightarrow X$ is a map. Then $f$ can be approximated by a map $f^{\prime}$ such that $\mathcal{C} \ell\left(S_{f}\right)$ is a tame, compact subset of $M$ of dimension $\leq 2 n-m, f(M)$ is a 1-LCC compactum in $X$ of dimension $\leq m$, and $\operatorname{dim}\left(f^{\prime}\left(\mathcal{C} \ell\left(S_{f^{\prime}}\right)\right)\right) \leq 2 n-m$.

Proof. Given $\epsilon>0$, choose $\delta>0$ and $\mu>0$ as in Corollary 2.3. Use Theorem 2.9 to get $f$ in general position. Set $S=S_{f}, Z=f(S)$, and, for any $r>0$, let $Z_{r}=\left\{z \in Z: \operatorname{diam} f^{-1}(z) \geq r\right\}$ and $S_{r}=f^{-1}\left(Z_{r}\right)$. Let $S^{\prime}=\mathcal{C} \ell\left(f^{-1}\left(Z_{\delta / 2} \backslash Z_{\delta / 3}\right) \cap\right.$ $f^{-1}\left(Z_{\delta / 3}\right)$ ), and let $Z^{\prime}=f\left(S^{\prime}\right)$. In general, $S^{\prime}$ might not be a complete inverse set. Now apply the Main Construction to eliminate the singularities in $S^{\prime}$. The resulting map $f^{\prime}: M \rightarrow X$ will be $\epsilon$-homotopic to $f$, and $\mathcal{C} \ell\left(S_{f^{\prime}}\right)$ is the image of $S_{\delta / 2}$ under a map from $M$ to $M$ that shrinks the mapping cylinder cones over $S^{\prime}$. Hence, $\mathcal{C} \ell\left(S_{f^{\prime}}\right)$ is a compact set in $M$ of dimension $\leq 2 m-n$ whose image under $f^{\prime}$ also has dimension $\leq 2 m-n$. Tameness properties follow from the constructions.

Theorem 5.2. Suppose that $X^{n}, n \geq 5$, is an $n$-gm with the $D D P, M^{m}$ is a compact $P L$ m-manifold, $3 m \leq 2 n-2$, and $f: M \rightarrow X$ is a map. Then $f$ can be approximated by a map $f^{\prime}$ such that

(1) $\mathcal{C} \ell\left(S_{f^{\prime}}\right)$ is a tame, compact polyhedron in $M$, of dimension $\leq 2 m-n$,

(2) $f(M)$ is a 1-LCC subset of $X$,

(3) $S_{f^{\prime}}$ consists only of double-points,

(4) $\mathrm{Br}=\mathcal{C} \ell\left(S_{f^{\prime}}\right) \backslash S_{f^{\prime}}$, the branched set of $f^{\prime}$, is a polyhedron of dimension $\leq 2 m-n-1$,

(5) $f^{\prime} \mid M \backslash B r$ is a local homeomorphism, and

(6) $f^{\prime} \mid B r$ is one-to-one.

Proof. By Lemma 5.1 we may assume that $\mathcal{C} \ell\left(S_{f}\right)$ is a compactum of dimension $\leq 2 m-n$ tamely embedded in $M$ and that $f(M)$ is 1-LCC in $X$ and has dimension $\leq m$. An application of general position, given our dimension restrictions, allows us to assume that $f$ has at most double-point singularities and that $n \geq 6$.

Our first step is to adjust $f$ so that $S_{f}$ is open in $\mathcal{C} \ell\left(S_{f}\right)$. Set $S=S_{f}$ and $Z=f(S)$. Given $\epsilon>0$, let $Z_{\epsilon}=\left\{z \in Z: \operatorname{diam} f^{-1}(z)>\epsilon\right\}$, and let $S_{\epsilon}=f^{-1}\left(Z_{\epsilon}\right)$. (This use of the notation $Z_{\epsilon}$ and $S_{\epsilon}$ is slightly different than that used in previous arguments.) Given $z_{0} \in Z_{\epsilon}$, choose $\epsilon_{0}>0$ such that if $f^{-1}\left(z_{0}\right)=\left\{x_{0}, x_{0}^{\prime}\right\}$, then $d\left(x_{0}, x_{0}^{\prime}\right) \geq \epsilon+\epsilon_{0}$. There is a neighborhood $U_{0}$ of $z_{0}$ in $X$ such that if $z \in Z \cap$ $U_{0}$, then $f^{-1}(z)$ lies in the $\left(\epsilon_{0} / 2\right)$-neighborhood of $f^{-1}\left(z_{0}\right)$. This implies that, if $f^{-1}(z)=\left\{x, x^{\prime}\right\}$, then $\left\{x, x^{\prime}\right\}$ lies in the $\left(\epsilon_{0} / 2\right)$-neighborhood of $x_{0}$ or in the $\left(\epsilon_{0} / 2\right)$ neighborhood of $x_{0}^{\prime}$. Thus, for any positive function $\delta$ on $Z_{\epsilon}$, we can find an open 
set $U$ in $X$ containing $Z_{\epsilon}$ such that if $z \in Z \cap U$, then $f^{-1}(z)$ lies in the $\delta\left(z_{0}\right)$ neighborhood of $x_{0} \in f^{-1}\left(z_{0}\right)$ for some $z_{0} \in Z_{\epsilon}$. We can then apply the Main Construction to approximate $f$ by a map $f^{\prime}$ with no singularities in $\left(f^{\prime}\right)^{-1}(U)$ that are not in $S_{\epsilon}$. We will then have that $S_{\epsilon}$ is open in $\mathcal{C} \ell\left(S_{f^{\prime}}\right)$. Applying this procedure inductively over the sets $Z_{1 / k}, k=1,2, \ldots$, we arrive at an approximation $f^{\prime}$ of $f$ with the desired property.

Assume, then, that $f$ has the property that $S$ is open in $\mathcal{C} \ell(S)$. Let $Z_{0}=\mathcal{C} \ell Z \backslash Z$ and $S_{0}=f^{-1}\left(Z_{0}\right)$, the branched set of $f$. Since $f$ has at most double-point singularities, $f \mid S_{0}: S_{0} \rightarrow Z_{0}$ is one-to-one. We also have that $f \mid M \backslash Z_{0}$ is a local homeomorphism so that $f \mid S: S \rightarrow Z$ is a double cover.

The plan now is to momentarily isolate $S_{0}$ by an (inverse) open set $V_{0}$ and to adjust $f$ so that the singularities in $S \backslash V_{0}$ form a polyhedron that maps to a polyhedron in $X$. We will then eliminate the singularities in $S \cap V_{0}$, producing a new polyhedral branch set of the predicted dimension.

For a given $\delta>0$, let $U_{0}$ be an open set in $X$ such that $Z_{0} \subseteq U_{0}, \operatorname{diam} f^{-1}(z)<\delta$, and bd $U_{0} \cap Z$ has dimension $\leq 2 m-n-1$. Set $V_{0}=f^{-1}\left(U_{0}\right), Z_{1}=Z \backslash U_{0}, S_{1}=$ $f^{-1}\left(Z_{1}\right)$, and $k=2 m-n$. Let $\mathcal{U}$ be a fine open cover of $Z_{1}$ of order $\leq k+1$ whose restriction to $\operatorname{bd}_{Z} Z_{1}=\operatorname{bd} U_{0} \cap Z$ has order $\leq k$, chosen so that for each $U \in \mathcal{U}$, $f^{-1}(U)=V_{1} \cup V_{2}$, where $F \mid V_{i}: V_{i} \rightarrow U \cap f(M)$ is a homeomorphism. Let $\mathcal{V}=$ $f^{-1}(\mathcal{U}) . \mathcal{V}$ is a cover of $S_{1}$ of order $\leq k+1$ whose restriction to $\operatorname{bd}_{S} S_{1}=f^{-1}\left(\operatorname{bd}_{Z} Z_{1}\right)$ has order $\leq k$. Then $f \mid \bigcup \mathcal{V}: \bigcup \mathcal{V} \rightarrow \bigcup \mathcal{U}$ is a double cover. Moreover, one can construct nerves $\left(P, P_{0}\right)$ of $\left(\mathcal{V}, \mathcal{V} \mid \mathrm{bd}_{S} S_{1}\right)$ and $\left(Q, Q_{0}\right)$ of $\left(\mathcal{U}, \mathcal{U} \mid \mathrm{bd}_{Z} Z_{1}\right)$ that admit a double cover $p:\left(P, P_{0}\right) \rightarrow\left(Q, Q_{0}\right)$. Working inductively over skeleta of $P$ and $Q$, we can construct small maps $\phi:\left(S_{1}, \operatorname{bd}_{S} S_{1}\right) \rightarrow\left(P, P_{0}\right)$ and $\psi:\left(Z_{1}, \operatorname{bd}_{Z} Z_{1}\right) \rightarrow\left(Q, Q_{0}\right)$ such that $p \circ \phi=\psi \circ f$.

Perform the following construction inductively over $Q$ : Let $\sigma$ be a simplex of $Q$ with $p^{-1}(\sigma)=\sigma_{1} \cup \sigma_{2}$, where $\sigma_{i}$ is a simplex in $P, i=1,2$. Assume that $\phi \mid \phi^{-1}\left(\operatorname{bd} \sigma_{i}\right): \phi^{-1}\left(\operatorname{bd} \sigma_{i}\right) \rightarrow \operatorname{bd} \sigma_{i}, i=1,2$, and $\psi \mid \psi^{-1}(\operatorname{bd} \sigma): \psi^{-1}(\operatorname{bd} \sigma) \rightarrow \operatorname{bd} \sigma$ are the identity maps. (That is, we are assuming that the singular set agrees with a skeleton of $P$.) Set $\left(A_{i}, \dot{A}_{i}\right)=\phi^{-1}\left(\sigma_{i}\right.$, bd $\left.\sigma_{i}\right), i=1,2$, and $(B, \dot{B})=\psi^{-1}(\sigma, \operatorname{bd} \sigma)$. Then $f \mid A_{i}$ maps $\left(A_{i}, \dot{A}_{i}\right)$ homeomorphically onto $(B, \dot{B})$. Let $C_{i}$ be the reduced mapping cylinder of $\phi \mid A_{i}: A_{i} \rightarrow \sigma_{i}\left(\bmod \dot{A}_{i}\right)$ with projection $\gamma_{i}: C_{i} \rightarrow \sigma_{i}$. If we have been careful so far, then we can get tame embeddings of $C_{i} \subset M$, extending the inclusion on $A_{i}$ so that mapping cylinder fibers are small and so that $C_{i} \cap \mathcal{C} \ell S=A_{i}$ and $C_{1} \cap C_{2}=\varnothing$. We can assume further that $C_{1}$ and $C_{2}$ lie in disjoint open sets $V_{1}$ and $V_{2}$, respectively, such that $f \mid V_{i}: V_{i} \rightarrow f\left(V_{i}\right)$ is a homeomorphism and that $f\left(C_{1}\right) \cap f\left(C_{2}\right)=\sigma$. Thicken $f\left(V_{1}\right)$ to an open set $U_{1}$ in $X$. Shrink the mapping cylinder $C_{1}$ to $\sigma_{1}$ in $M$ and homotopically shrink $f\left(C_{1}\right)$ to $\sigma$ in $X$ with a strict homotopy $H_{t}, t \in I$, of $\left(U_{1}, f\left(V_{1}\right)\right)$ having compact support. This homotopy will drag $f\left(V_{2}\right)$ along the fibers of $f\left(C_{1}\right)$, but will not create any new intersections of $H_{t} \circ f \mid V_{2}$ with $f\left(V_{1}\right)$. Thus, we can ensure that the only new nondegenerate point-inverses in $H_{1} \circ f \mid V_{2}$ are the point-inverses of $\phi_{2}$ and small sets, each of which lies near a fiber of $C_{2}$. The latter type may be eliminated using the Main Construction. To eliminate the point-inverses of $\phi_{2}$, get a 1-LCC embedding of the reduced mapping cylinder $D_{2}$ of $\gamma_{2}: A_{2} \rightarrow \sigma_{2}\left(\bmod A_{2}\right)$ into $X$ so that its intersection with $H_{1} \circ f(M)$ is $H_{1} \circ f\left(C_{2}\right)$. Now shrink the fibers of $C_{2}$ in $M$ and homotopically shrink the fibers of $D_{2}$ in $X$, eliminating any newly introduced singularities using the Main Construction. (This homotopy shrinking, unlike the 
first one, is not required to move points of $Z$; hence, the only new singularities will lie in $V_{2}$ and consist of small double points.)

This process leads to an approximation of $f$, which we will still call $f$, such that, for some $\epsilon>0, f\left(\mathcal{C} \ell\left(S_{f}\right)\right)=Z_{1} \cup Z_{2}, \mathcal{C} \ell\left(S_{f}\right)=S_{1} \cup S_{2}$, where $Z_{i}$ is closed in $X$ and $S_{i}=f^{-1}\left(Z_{i}\right), i=1,2$, such that

(a) $S_{1}$ is a tame polyhedron in $M$ and $Z_{1}$ is a 1-LCC polyhedron $X$ of dimensions $\leq 2 m-n$

(b) $f(M)$ is 1 -LCC in $X$,

(c) $f \mid S_{1}: S_{1} \rightarrow Z_{1}$ is a double cover,

(d) $Z_{1} \cap Z_{2}$ and $S_{1} \cap S_{2}$ are polyhedra of dimension $\leq 2 m-n-1$, and

(e) $\operatorname{diam} f^{-1}(z)<\epsilon$, for all $z \in Z_{2}$.

If $\epsilon$ is sufficiently small, we may use the Main Construction again to eliminate the singularities over $Z_{2}$, producing a branched set homeomorphic to $Z_{1} \cap Z_{2}$.

As a final application of the Main Construction, we can establish a Whitney trick for getting $P L$ submanifolds of a generalized manifold of complementary dimensions to be disjoint when the homology conditions are right. Suppose that $X$ is an oriented $n$-gm with the $D D P, n \geq 5$, and that $M$ is a closed, oriented topological submanifold of $X$. Let $V \subset M$ be an open $m$-ball neighborhood of a point $x \in M$, and let $U$ be an open set in $X$ such that $U \cap M=V$. Then

$$
H_{n-p}(U, U \backslash V) \cong \tilde{H}_{c}^{p}(V) \cong \begin{cases}\mathbb{Z}, & \text { if } p=m, \\ 0, & \text { otherwise. }\end{cases}
$$

Suppose that $q=n-m$ and that $Q$ is an oriented, $q$-dimensional (topological) submanifold of $X$ such that $Q \cap V=\{x\}$. Let $W=Q \cap U$. Then $H_{q}(W, W \backslash\{x\}) \cong$ $\mathbb{Z}$. Thus, the inclusion $H_{q}(W, W \backslash\{x\}) \rightarrow H_{q}(U, U \backslash V)$ is multiplication by an integer $k$, which we define to be the intersection number of $M$ and $Q$ at $x$. If $M \cap Q$ is a finite set $F$, define the intersection number of $M$ and $Q$ to be the sum over $F$ of the intersections numbers at $x \in F$.

Theorem 5.3. Suppose that $X^{n}, n \geq 6$, is an orientable $n$-gm with the DDP, $M^{m}$ and $Q^{q}$ are compact orientable $P L$ manifolds, tamely embedded in $X$, with $3 \leq m \leq q \leq n-3$ and $m+q=n$. Then for every $\epsilon>0$ there is an $\epsilon$-homotopy of $M$ in $X$ to a tame embedding $f: M \rightarrow X$ such that $f(M)$ is transverse to $Q$. That is, $f(M) \cap Q$ is a finite set $F$, and the intersection number of $f(M) \cap Q$ at each point of $F$ is \pm 1 .

Proof. Apply Theorem 2.9 to get a homotopy of the inclusion of $M$ in $X$ to a map $f: M \rightarrow X$ so that $f(M) \cap Q$ is a compact, 1-LCC 0 -dimensional set $F$ in $Q$ and $f^{-1}(Q)$ is a compact, 1 -LCC 0 -dimensional set in $M$. Our dimension restrictions allow us to assume that $f\left(S_{f}\right) \cap Q=\varnothing$. Since $2 m \leq n$, we can apply the Main Construction to eliminate the singularities of $f$ by a homotopy supported in a neighborhood of a tame set of dimension $\leq 2 m-n+2$ in $X$ (the mapping cylinders we labeled $D$ in the Main Construction). Our dimension restrictions imply that these sets can be made to miss $Q$, by Theorem 2.9. Thus, we may assume that $M \cap Q$ is a compact, 1-LCC 0 -dimensional set in both $M$ and $Q$. We first reduce $F$ to a finite set.

Suppose that $G$ is a small, open-closed subset of $F$. Get a small $m$-ball $B$ in $M$ and a small $q$-ball $D$ in $Q$ such that $B \cap F=\operatorname{int} B \cap F=G$ and $D \cap F=\operatorname{int} D \cap F=$ $G$. Let $U$ be a small open set in $X$ such that $U \cap M=\operatorname{int} B$ and $U \cap Q=\operatorname{int} D$. Let 
$A$ be a tame arc in int $D$ such that $A \cap F=G$. Let $h_{t}, t \in I$, be a pseudo-isotopy of $Q$, supported on a compact set in int $D$, that shrinks $A$ to a point $x$ in $A$. Since $Q$ is 1-LCC in $X$, we can apply Lemma 2.8 to get a homotopy $H_{t}, t \in I$, of $U$ with compact support such that $H_{0}=\mathrm{id}, H_{t} \mid Q=h_{t}$, and $H_{t}(U \backslash D) \subset(U \backslash D)$ for all $t \in I$.

The homotopy $H_{t}$, restricted to $M$, is a homotopy of $M$ in $X$ such that $H_{1}(B) \cap$ $Q=\{x\}$. As above, we can eliminate the singularities of $H_{1} \mid M$ by a homotopy that does not introduce any new intersections with $Q$.

Applying this construction to a finite cover of $F$ by small, mutually exclusive open-closed sets gives a small homotopy of $M$ in $X$ to a 1-LCC embedding $f: M \rightarrow$ $X$ such that $f(M) \cap Q$ is a finite set. So assume that $M \cap Q=F$ is finite.

Suppose that $x \in F$ and the intersection number $M \cap Q$ is 0 at $x$. Let $V$ be a small open $q$-ball around $x$ in $Q$. Since $Q$ is tame in $X$, there are small open sets $U_{1} \subset U_{2} \subset \cdots \subset U_{\ell}$ in $X$ such that $U_{i} \cap Q=V$ and $U_{i}$ contracts to a point in $U_{i+1}$. Choose $\ell$ to satisfy the Eventual Hurewicz Theorem [8] so that any nullhomologous $(m-1)$-cycle in $U_{1} \backslash V$ is null-homotopic in $U_{\ell} \backslash V$. Let $B$ be a small $m$-ball in $M$, containing $x$ in its interior, lying in $U_{1}$, with boundary $S$. Then $S$ is null-homologous in $U_{1} \backslash V$, hence, null-homotopic in $U_{\ell} \backslash V$. This allows us to get a small homotopy of $M$ in $X$, supported in $B$, to a map of $f: M \rightarrow X$ such that $f(B) \cap Q=\varnothing$. Eliminate the singularities of $f$, using the Main Construction, without introducing new intersections with $Q$.

Suppose that $x \in F$ and the intersection number $M \cap Q$ is $r$ at $x,|r|>1$. Without loss of generality, assume $r>0$. As above, let $V$ be a small open $q$ ball around $x$ in $Q$. Choose small open sets $U_{1} \subset U_{2} \subset U_{3}$ in $X$ such that $U_{i} \cap$ $Q=V, U_{i}$ is contractible to a point in $U_{i+1}$, and each null-homologous ( $m-$ 1)-cycle in $U_{i} \backslash Q$ is null-homotopic in $U_{i+1} \backslash Q$. Let $B$ be a small $m$-ball in $M$, containing $x$ in its interior and lying in $U_{1}$. Use local duality in $X$ and the Eventual Hurewicz Theorem to get $r$ small, mutually exclusive, singular $m$-cells $B_{1}, \ldots, B_{r}$, with boundaries $S_{1}, \ldots, S_{r}$, such that $\left(B_{i}, S_{i}\right) \subset\left(U_{1}, U_{1} \backslash V\right)$ and such that the intersection number of $B_{i}$ and $U$ is 1 . Let $B_{1}^{\prime}, \ldots, B_{r}^{\prime}$ be mutually exclusive $m$-balls in int $B$ with boundary spheres $S_{1}^{\prime}, \ldots, S_{r}^{\prime}$, and let $f_{i}:\left(B_{i}^{\prime}, S_{i}^{\prime}\right) \rightarrow$ $\left(B_{i}, S_{i}\right)$ be appropriate maps. In $U_{2}, S$ is homologous to $\sum S_{i}$, hence, in $U_{3}$ there is an extension of $\bigcup\left(f_{i} \mid S_{i}\right): \bigcup S_{i} \rightarrow\left(U_{2} \backslash V\right)$ and the inclusion on $S$ to a map $\left(B \backslash \bigcup \operatorname{int} B_{i}\right) \rightarrow\left(U_{3} \backslash V\right)$. Extend over $\bigcup B_{i}$ by $\bigcup f_{i}$. We may eliminate the singularities of this map to get a tame embedding $f: M \rightarrow X$ that agrees with the inclusion outside a neighborhood of $x$ such that $f(M) \cap Q \subset \bigcup f\left(B_{i}\right)$. Apply the argument at the beginning of this proof to get $f\left(B_{i}\right) \cap Q$ to be a single point for each $i=1, \ldots, r$, but otherwise introducing no new intersections in $f(B) \cap Q$.

Theorem 5.4. Suppose that $X^{n}, n \geq 6$, is a simply connected $n$-gm with the DDP, $M^{m}$ and $Q^{q}$ are compact, connected, orientable PL m-manifolds, tamely embedded in $X, 3 \leq m \leq q \leq n-3$ and $m+q=n$, with intersection number 0 . Then there is a homotopy of $M$ in $X$, supported on a neighborhood of a mutually exclusive family of 2-cells in $X$, to a tame embedding $f: M \rightarrow X$ such that $f(M) \cap Q=\varnothing$.

Proof. By Theorem 5.3 we may assume that $M \cap Q$ is a finite set $F$ and that $M \cap Q$ has intersection number \pm 1 at each point of $F$. The proof now follows the classical line of argument. Since $M$ and $Q$ have intersection number 0 , we can pair the points of $F$, say $x$ with $y$ in $F$, so that $M \cap Q$ has intersection number 1 at $x$ and -1 at $y$. 
Choose tame $\operatorname{arcs} A \subset M$ and $A^{\prime} \subset Q$, each with endpoints $x$ and $y$, so that $A \cap F=A^{\prime} \cap F=\{x, y\}$. Let $D$ be a 1-LCC 2-cell in $X$ such that $D \cap(M \cup Q)=$ $A \cup A^{\prime}$. Apply Lemma 2.8 to get a homotopy $H_{t}$ of $X, t \in I$, supported in a neighborhood of $A^{\prime}$ in $X$, such that $H_{0}=\mathrm{id}, H_{t} \mid Q$ is a pseudo-isotopy of $Q$ that shrinks $A^{\prime}$ to $x$, and $H_{t}(X \backslash Q) \subset(X \backslash Q)$ for all $t \in I$. Without loss of generality, $H_{1}(D)$ is a 1-LCC 2-cell in $X$ such that $H_{1}(D) \cap(M \cup Q)=H_{1}(A)$. Set $f=$ $H_{1} \mid M$. Eliminate the singularities of $f$ except $x$ and $y$ without introducing further intersections with $Q$, using the the Main Construction. Let $D^{\prime}=H_{1}(D)$. Use $D^{\prime}$ to eliminate the singularity $f(x)=f(y)$ without introducing further intersections with $Q$. This produces a homotopy of $M$ in $X$ supported in $D$ to a 1-LCC embedding $g: M \rightarrow X$ with intersection number 0 at $x$. Eliminate this intersection using the argument from the proof of Theorem 5.3.

\section{REFERENCES}

[1] J. L. Bryant, On embeddings of compacta in Euclidean space, Proc. Amer. Math. Soc. 23 (1969), 46-51. MR 39:6286

[2] — General position theorems for generalized manifolds, Proc. Amer. Math. Soc. 98 (1986), 667-670. MR 87m:57020

[3] J. Bryant, S. Ferry, W. Mio, and S. Weinberger, Topology of homology manifolds, Ann. of Math.(2) 143 (1996), 435-467 MR 97b:57017

[4] J. W. Cannon, The recognition problem: what is a topological manifold?, Bull. Amer. Math. Soc. 84 (1978), 832-866. MR 58:13043

[5] _ Shrinking cell-like decompositions of manifolds in codimension three, Ann. of Math.(2) 110 (1979), 83-112. MR 80j:57013

[6] R. J. Daverman, Decompositions of Manifolds, Pure and Applied Math., 124, Academic Press, Orlando, Florida, 1986. MR 88a:57001

[7] R. D. Edwards, Dimension theory, Lecture Notes in Mathematics, Vol. 438, Springer-Verlag, Berlin, 1975. MR 52:15477

[8] S. Ferry, Homotoping e-maps to homeomorphisms, Amer. J. Math. 101 (1979), 567-582. MR 81f:57007a

[9] J. F. P. Hudson, Piecewise linear embeddings, Ann. of Math. 85 (1967), 1-31. MR 35:6149

[10] W. Hurewicz, and H. Wallman, Dimension Theory. Princeton Univ. Press, Princeton, New Jersey, 1941. MR 3:312b

[11] R. Kirby and L. Siebenmann, Foundational Essays on Topological Manifolds, Smoothings, and Triangulations, Princeton University Press, Princeton, N.J., 1977. MR 58:31082

[12] A. Nicas, Induction Theorems for groups of manifold structure sets, Mem. Amer. Math. Soc., vol. 39, 1982. MR 83i:57026

[13] F. Quinn, Resolutions of homology manifolds, the topological characterization of manifolds, Invent. Math. 72 (1983), 267-284. MR 85b:57023; Corrigendum, MR 87g:57031

[14] _ An obstruction to the resolution of homology manifolds, Mich. Math. J. 34 (1987), 285-292. MR 88j:57016

[15] — Homotopically stratified sets, J. Amer. Math. Soc. 1 (1988), 441-499. MR 89g:57050

[16] J. Stalling, The embedding of homotopy types into manifolds, Unpublished.

[17] M. A. Stan'ko, The embedding of compacta in Euclidean space, Mat. Sb. 83 (125) (1970), 234-255; Math. USSR-Sb. 12 (1970), 234-254. MR 42:6804

[18] M. A. Štan'ko, Approximation of compacta in $E^{n}$ in codimension greater than two, Mat. Sb. 90 (132) (1973), 625-636; Math. USSR-Sb. 19 (1973), 615-626. MR 49:3957

[19] C. T. C. Wall, Surgery on Compact Manifolds, Academic Press, New York, 1970. MR 55:4217

[20] J. Walsh, General position properties of generalized manifolds: a primer, Proc. Idaho State Topology Conf., 1985.

Department of Mathematics, Florida State University, Tallahassee, Florida 32306

E-mail address: bryant@math.fsu.edu

E-mail address: mio@math.fsu.edu 